\title{
Are food record charts useful components of nutritional assessment?
}

\author{
T. Fujiwara, M. Cartlidge and R. A. Richardson \\ Dietetic Service, NHS Greater Glasgow Adult Acute Division, Southern General Hospital, Glasgow G51 4TF, UK
}

Food record charts (FRC) are routinely used to monitor and assess the oral intake of patients deemed to be at nutritional risk and are normally completed by nursing staff. Subsequently, they are used to inform and direct dietetic treatment plans. However, there is little evidence on the validity of the qualitative and quantitative information recorded in FRC. An audit, in two stages, to examine the information recorded was undertaken to determine the strength of this methodology in examining antecedent dietary intake.

Stage 1 focused on appraising the quality of information recorded on FRC. Each FRC was arbitrarily scored out of ten, with two points assigned for detailed recording at each mealtime (breakfast, lunch and evening meal) and one point for snacks (mid morning, mid afternoon and supper). A further discretionary point was awarded if the chart clearly identified the types of food and fluids consumed at two or more mealtimes.

Stage 2 examined patients' estimated energy intake derived from FRC for one meal (lunch) in twenty randomly-selected patients and compared this intake with that from weighed intake for the same meal (WISP; Tinuviel Software, Warrington, UK). To eliminate bias one dietitian was responsible for analysis of FRC and another undertook the weighed intake component. Results are expressed as means with their standard errors and a Wilcoxon's signed ranks test was used in pair-wise comparisons and Spearman's rank correlation to examine the association between variables.

None of the twenty FRC was completed to the extent that they provided sufficient information for full interpretation (mean score 5 (range 0-9) of 10). There was no significant difference between energy intake estimated from FRC or weighed intake for the one meal studied (estimated intake 1071 (SE 118) kJ; measured intake 1310 (SE 127) kJ). There was, however, no association between estimated and actual energy intake $(r 0.42)$ at this meal.

Whilst no differences in energy intake derived by either method was observed, this result is at odds with the poor completion rate of FRC and the lack of association between FRC and weighed intake. The lack of consistency in the statistical results may be due to the relatively small patient numbers who were studied at only one meal episode. The rationale for the study design was partly dictated by available resources to undertake this work. Nevertheless, this small study has acted to direct future work in this area, where undertaking a more robust study (including power calculation) would add value to the process. The incompleteness of FRC completed by staff is an area of immediate concern, but with the imminent implementation of Food, Fluid and Nutritional Care in Hospitals ${ }^{(1)}$ this process may be timely in terms of re-evaluation.

1. NHS Quality Improvement Scotland (2003) Food, fluid and nutritional care in hospitals. http://www.nhshealthquality.org/nhsqis/files/Food, $\% 20$ Fluid\%20Nutrition.pdf 